\title{
NUCLEATION, KINETICS AND ADMISSIBILITY CRITERIA FOR PROPAGATING PHASE BOUNDARIES
}

\author{
ROHAN ABEYARATNE* AND JAMES K. KNOWLES †
}

\begin{abstract}
This paper reviews our recent studies on the nucleation and kinetics of propagating phase boundaries in an elastic bar and relates them to various admissibility criteria. First, we discuss how the field equations and jump conditions of the quasi-static theory of such a bar must be supplemented with additional constitutive information pertaining to the initiation and evolution of phase boundaries. The kinetic relation relates the driving traction $f$ at a phase boundary to the phase boundary velocity $\dot{s}$; thus $f=\varphi(\dot{s})$, where $\varphi$ is a materially-determined function. The nucleation criterion specifies a critical value of $f$ at an incipient phase boundary. We then incorporate inertial effects, and we find in the context of the Riemann problem that, as long as phase boundary velocities are subsonic, the theory again needs - and has room for - a nucleation criterion and a kinetic relation. Finally, we describe the sense in which each of three widely studied admissibility criteria for phase boundaries is equivalent to a specific kinetic relation of the form $f=\varphi(\dot{s})$ for a particular choice of $\varphi$. A kinetic relation based on thermal activation theory is also discussed.
\end{abstract}

1. Introduction. In the simplest theory of longitudinal motions of an elastic bar, one considers a pair of conservation laws in one space dimension for the strain $\gamma(x, t)$ and the particle velocity $v(x, t)$ at time $t$ and position $x$. If there is a strain discontinuity at $x=s(t)$, it is subject to a pair of jump conditions associated with the conservation laws. In addition, it is required that the entropy of a particle must not decrease as it crosses such a discontinuity; we refer to this condition as the entropy inequality.

The character of the material of the bar enters the differential equations and jump conditions through the relation $\sigma=\widehat{\sigma}(\gamma)$ between the stress $\sigma(x, t)$ and the strain. If the material is such that $\widehat{\sigma}(\gamma)$ is a monotonically increasing function that is either strictly convex or strictly concave, strain discontinuities are shock waves, and as in gas dynamics, the entropy inequality serves to single out a piecewise smooth solution to the Cauchy problem. A uniqueness theorem that pertains to this situation was given by Oleinik [28]; Liu [26] discusses Oleinik's theorem and related results.

If one wishes to employ the theory described above to construct a simple, purely mechanical continuum model of stress-induced solid-solid phase transitions, one must relinquish the requirement of non-vanishing curvature of the stress-strain curve; indeed, if equilibrium phase mixtures are to be included in the phenomena described by the model, then the monotonicity of $\widehat{\sigma}(\gamma)$ must be given up as well. Thus in such theories of phase transformations, one typically encounters stress response functions $\widehat{\sigma}(\gamma)$ in which stress at first increases with increasing strain, then 
each of the three branches of such a stress-strain curve with a "phase" of the material; the declining branch is associated with the unstable phase. A propagating discontinuity is a shock wave if the strains on the two sides of the jump belong to the same phase; otherwise, the discontinuity is a phase boundary.

For a stress-strain curve that is non-monotonic and undergoes a change in the sign of its curvature, the entropy inequality alone is no longer sufficient to deliver uniqueness in the Cauchy problem. Indeed, as shown in [1-3], lack of uniqueness arises not only in dynamics, but in the theory of phase transitions in quasi-static motions as well. In [2], we viewed the lack of determinacy in quasi-statics as reflecting the need to incorporate in the continuum model a nucleation criterion and a kinetic relation to control the initiation and evolution, respectively, of the phase transition. The inclusion of these two additional pieces of constitutive information leads to a determinate quasi-static theory whose predictions are in qualitative accord with experiments that involve slowly propagating phase boundaries in tension bars; see [2].

If the kinetic relation and the nucleation criterion are indeed part of the constitutive description of the material, they must apply not only in quasi-static motions, but when inertia is present as well. In [5], we have shown in the context of the Riemann problem for a special elastic material that the lack of uniqueness of solution remaining after imposition of the entropy inequality is precisely that needed to accommodate the nucleation criterion and the kinetic relation at subsonically propagating phase boundaries. We also showed in [5] that the kinetic relation cannot be prescribed at phase boundaries that propagate supersonically with respect to the phase with lower sound speed.

Other approaches to the issue of lack of uniqueness of solution to dynamical problems in the presence of phase transformations have typically involved the replacement of the entropy inequality by a stronger solution-selecting mechanism, or admissibility criterion. Among these, perhaps the most common are the viscositystrain gradient criterion, in which the elastic stress-strain law is modified by incorporating viscous and strain-gradient effects, the entropy rate shock admissibility criterion, which implements one notion of maximum dissipation, and the chord criterion, which is related to a second notion of maximum dissipation. For a special elastic material, we showed in [6] that the viscosity-strain gradient criterion is equivalent to a particular kinetic-relation of the type we had studied in [2] and [5]. In [7], we showed - again for a special material - that the same is true of the entropy rate shock admissibility criterion.

The objective of the present expository paper is to provide a unified review of the work reported in [2], [5], [6] and [7]. After setting out the fundamentals in Section 2, we describe in Section 3 the quasi-static theory developed in [2]. Section 4 summarizes the study of the dynamics of phase transitions carried out in [5]. Finally, 
of the bar, the particle located at $x$ in the reference configuration is carried to the point $x+u(x, t)$ at time $t$; the displacement $u$ is required to be continuous and to have piecewise continuous first and second derivatives on $[0, L] \times[0, \infty)$. At points $(x, t)$ in space-time where $u_{x}$ and $u_{t}$ exist, we let $\gamma=u_{x}, v=u_{t}$ denote strain and particle velocity, respectively. In order to assure that the mapping $x \rightarrow x+u(x, t)$ is invertible at each $t$, the strain $\gamma(x, t)$ is required to exceed -1 . The stress $\sigma(x, t)$ is related to the strain through $\sigma=\widehat{\sigma}(\gamma)$, where the stress response function $\widehat{\sigma}$ is determined by the material. The mass density $\rho$ in the reference configuration is taken to be constant.

Balance of linear momentum and the smoothness properties of $u$ require that, at points where $\gamma$ and $v$ are smooth,

$$
\begin{gathered}
\hat{\sigma}^{\prime}(\gamma) \gamma_{x}-\rho v_{t}=0 \\
v_{x}-\gamma_{t}=0
\end{gathered}
$$

if either $\gamma$ or $v$ jumps across the curve $x=s(t)$ in the $x, t$-plane, the balance laws also require that

$$
\begin{aligned}
& \stackrel{+}{\sigma}-\bar{\sigma}=-\rho \dot{s}(\stackrel{+}{v}-\bar{v}) \\
& (\stackrel{\gamma}{\gamma}-\bar{\gamma}) \dot{s}=-(\stackrel{+}{v}-\bar{v})
\end{aligned}
$$

where for any function $g(x, t)$, we write $\stackrel{+}{g}=g(s(t)+, t), \bar{g}=g(s(t)-, t)$.

Even though the bar is elastic, the basic field equations and jump conditions (2.1)-(2.4) do not guarantee that the instantaneous dissipation rate during a motion is non-negative. In order to assure this, one must impose an additional requirement at each strain discontinuity. To this end, we let $W(\gamma)$ be the referential strain energy density for the material: $W(\gamma)=\int_{0}^{\gamma} \widehat{\sigma}\left(\gamma^{\prime}\right) d \gamma^{\prime}$. Consider the restriction of the motion to the time interval $\left[t_{1}, t_{2}\right]$ and to the piece of the bar that occupies the interval $\left[x_{1}, x_{2}\right]$ in the reference configuration, and suppose that $\gamma$ and $v$ are smooth on $\left[x_{1}, x_{2}\right] \times\left[t_{1}, t_{2}\right]$ except at the moving strain discontinuity $x=s(t)$. The difference between the rate of work of the forces external to the piece of the bar under consideration and the rate of change of the associated total energy is the rate of dissipation $D(t)$ of mechanical energy: $D(t)=\sigma\left(x_{2}, t\right) A v\left(x_{2}, t\right)-\sigma\left(x_{1}, t\right) A v\left(x_{1}, t\right)-$ $\dot{E}(t)$, where $E(t)$ is the sum at time $t$ of the kinetic and strain energies in $\left[x_{1}, x_{2}\right]$. A direct calculation using (2.1)-(2.4) establishes the following alternative expression for the dissipation rate in terms of local quantities at the discontinuity:

$$
D(t)=f(t) A \dot{s}(t)
$$


and $\stackrel{+}{\gamma}, \bar{\gamma}$ are the strains on the two sides of the discontinuity. If there is no jump in strain at $x=s(t),(2.6)$ shows that $f=0$, and so $D$ vanishes in this special circumstance. Since

$$
\sigma\left(x_{2}, t\right) A v\left(x_{2}, t\right)-\sigma\left(x_{1}, t\right) A v\left(x_{1}, t\right)+(-f(t)) A \dot{s}(t)=\dot{E}(t)
$$

we may view $-f(t) A \dot{s}(t)$ as the rate of work done on the bar by the moving discontinuity. We therefore refer to $f$ as the driving traction, $f A$ as the driving force, exerted by the bar on the discontinuity; $f$ is related to the notion of the "force on a defect" introduced in a more general context by Eshelby [15, 16] and discussed by one of the present authors [23] and by Rice [35]. According to (2.6), the driving traction $f$ may be interpreted geometrically as the difference between the area under the stress-strain curve between the strains $\bar{\gamma}$ and $\stackrel{+}{\gamma}$ and the area of the trapezoid determined by $\bar{\gamma}, \stackrel{+}{\gamma}, \widehat{\sigma}(\stackrel{+}{\gamma})$, and $\widehat{\sigma}(\bar{\gamma})$.

In order to guarantee that the instantaneous dissipation rate associated with every piece of the bar is non-negative during the motion, one must enforce the additional requirement

$$
f(t) \dot{s}(t) \geq 0
$$

at each strain discontinuity and at all times. If the material is viewed as being thermoelastic, and if we make the assumption - however unrealistic - that the motion take place isothermally at a temperature $\theta$, then one can show that the rate of entropy production at time $t$ associated with the piece $\left[x_{1}, x_{2}\right]$ of the bar is $D(t) / \theta$; see [4]. Thus, under these conditions, the restriction (2.8) with $f$ given by $(2.6)$ is a consequence of the second law of thermodynamics; it is equivalent to the assertion that the entropy of a particle cannot decrease as the particle crosses a strain discontinuity. We shall refer to (2.8) as the entropy inequality.

If one wishes to allow for the possibility of stationary phase boundaries in a bar, it is necessary to consider a material whose stress response function $\widehat{\sigma}(\gamma)$ at first increases monotonically, then decreases, and finally increases again as $\gamma$ increases from the value $\gamma=-1$. In the present paper, we restrict attention to the special case in which $\widehat{\sigma}(\gamma)$ has the trilinear form shown in Figure 1; note that several material parameters are defined in the figure. Although many of our results apply only for this special material, some of them can be generalized to any rising-falling-rising stress-strain curve.

We shall say that a particle of the bar labeled by $x$ is in phase 1,2 or 3 at time $t$ according to whether the strain $\gamma(x, t)$ lies in the interval $\left(-1, \gamma_{M}\right],\left(\gamma_{M}, \gamma_{m}\right)$ or $\left[\gamma_{m}, \infty\right)$, respectively, corresponding to the three branches of the stress-strain curve in Fionre 1 In addition a strain discontinuity will he said to he of $n a$-tvne if $\bar{\gamma}$ 
Figure 1. Stress-strain curve for the trilinear material.

3. The quasi-static theory. The need for additional constitutive information in the presence of phase transitions manifests itself in both the quasi-static and the fully dynamic theories for the elastic bar. In this section, we outline the theory of quasi-static motions. A detailed discussion of this material is included in [2].

3.1 Equilibrium and quasi-static motions. For the trilinear material of Figure 1, we first seek equilibrium solutions of the field equations (2.1), (2.2) and jump conditions $(2.3),(2.4)$, corresponding to a displacement $u(x, t)$ that is independent of time $t$. In such solutions, the strain $\gamma$, the stress $\sigma$ and the discontinuity location $s$ are also independent of time, and the particle velocity $v$ vanishes. Conditions $(2.2),(2.4)$ are now trivially satisfied, and (2.1), (2.3) imply that $\sigma(x)=\sigma=$ constant for $0 \leq x \leq L$. For stress levels $\sigma$ between the local minimum $\sigma_{m}$ and the local maximum $\sigma_{M}$ in the stress-strain curve, it is possible to have equilibrium fields 
single discontinuity at $x=s$, where $s$ is given arbitrarily in $[0, L]$. An example of an equilibrium displacement field of this kind in which the strain to the left of $x=s$ is in phase 1 , while that to the right is in phase 3 , is given by

$$
u(x)=\left\{\begin{array}{l}
F x /\left(\mu_{1} A\right), \quad 0 \leq x \leq s, \\
F x /\left(\mu_{3} A\right)+\left(1 / \mu_{1}-1 / \mu_{3}\right) F s / A, \quad s \leq x \leq L ;
\end{array}\right.
$$

this field is said to be of 1,3-type. The relationship between the applied force $F$ and the overall elongation $\delta=u(L)-u(0)$ is called the macroscopic response of the bar. For the field of 1,3-type given by (3.1), the macroscopic response is

$$
\delta=\Delta_{13}(F, s) \equiv\left[\left(\frac{1}{\mu_{1}}-\frac{1}{\mu_{3}}\right) s+\frac{L}{\mu_{3}}\right] \frac{F}{A}, \quad \sigma_{m} A \leq F \leq \sigma_{M} A, 0 \leq s \leq L
$$

Next suppose that the given force $F$ lies in the interval $-\mu_{1} A<F \leq \sigma_{M} A$. Corresponding to such an $F$, there is a unique smooth equilibrium state in which the strain at every particle is in phase 1 . The associated macroscopic response is characterized by

$$
\delta=\Delta_{11}(F) \equiv F L /\left(\mu_{1} A\right), \quad-\mu_{1} A<F \leq \sigma_{M} A .
$$

Similarly, if $F \geq \sigma_{m} A$, there is a unique smooth equilibrium state in which the strain at every particle is in phase 3 , and the corresponding macroscopic response is

$$
\delta=\Delta_{33}(F) \equiv F L /\left(\mu_{3} A\right), \quad F \geq \sigma_{m} A .
$$

Observe that the mixed-phase displacement field (3.1) is smooth when either $s=L$ or $s=0$; in the former case, all particles of the bar are in phase 1 , while in the latter, the entire bar is in phase 3 . This is reflected by the fact that $\Delta_{11}(F)=\Delta_{13}(F, L)$ and $\Delta_{33}(F)=\Delta_{13}(F, 0)$ for $\sigma_{m} A \leq F \leq \sigma_{M} A$.

The macroscopic response for a field of 1,3-type is illustrated in Figure 2. The dashed lines represent graphs of $\delta=\Delta_{13}(F, s)$ vs. $F$ for various constant values of $s$. Points on $O P Q$ correspond to smooth phase- 1 fields with macroscopic response $\delta=\Delta_{11}(F)$; points on $S R T$ refer to smooth phase-3 fields, $\delta=\Delta_{33}(F)$. Points in the interior of the quadrilateral $P Q R S$ or on the horizontal portions of its boundary correspond to mixtures of phase 1 and phase 3 . Observe that, for a given force $F$ in $\left[\sigma_{m} A, \sigma_{M} A\right]$ or for a given elongation $\delta$ in $\left(\delta_{m}, \delta_{M}\right)$, there are infinitely many equilibrium states of 1,3-type, corresponding to a major breakdown of uniqueness.

The driving traction $f$ defined in (2.6), when specialized to the trilinear material and to an equilibrium 1,3-phase boundary, reduces to

$$
f=\frac{1}{2} \frac{\mu_{1}-\mu_{3}}{\mu_{1} \mu_{3}}\left(\sigma_{0}^{2}-\sigma^{2}\right)
$$

where $\sigma_{0}=\left(\sigma_{M} \sigma_{m}\right)^{1 / 2}$. The stress $\sigma_{0}$, which makes the hatched areas in Figure 1 
Figure 2. Macroscopic equilibrium states of 1,3-type.

A 1,3-quasi-static motion is obtained by replacing the force $F$ and the phase boundary location $s$ in (3.1) by functions of time: $F=F(t), s=s(t)$. The driving traction $f$ then becomes a function of time as well, and the entropy inequality (2.8), together with (3.5), yields

$$
\left[F(t)-\sigma_{0} A\right] \dot{s}(t) \leq 0
$$

as long as $\sigma_{m} A \leq F(t) \leq \sigma_{M} A$. A 1,3 -quasi-static motion determines a path in the quadrilateral $P Q R S$ in the force-elongation plane of Figure 2 ; the inequality 
at the phase boundary are transforming from phase 1 to phase 3 . The opposite conclusions apply when $F / A<\sigma_{0}$. When the stress $F(t) / A$ coincides with the Maxwell stress, one has $f=0$ and the entropy inequality (3.6) holds trivially; thus travel in either direction along the "Maxwell line" $F=\sigma_{0} A$ is possible, as indicated by the pertinent arrows in Figure 3.

Figure 3. Directions permitted by the entropy inequality (3.6) for paths of 1,3 -quasi-static motions.

In general, specifying the force history $F(\tau), 0 \leq \tau \leq t$, in a quasi-static motion is not sufficient to determine the present value $\delta(t)$ of the elongation, and vice-versa, even with the entropy inequality (2.8) enforced, since, according to (3.2), $\delta(t)$ depends on $s(t)$ as well. We view this lack of determinacy as arising from a constitutive deficiency in the theory, reflecting the need to specify two additional pieces of constitutive information pertaining to phase boundaries: a nucleation criterion 
3.2. Kinetic relations. For a 1,3-quasi-static motion, we shall postulate a simple form of kinetic relation in which there is a constitutive function $\varphi$ that relates the driving traction $f(t)$ at the phase boundary to the phase boundary velocity $\dot{s}(t)$ at each instant:

$$
f=\varphi(\dot{s}),-\infty<\dot{s}<\infty
$$

Because of the entropy inequality (2.8), the kinetic response function $\varphi$ is subject to the restriction

$$
\varphi(\dot{s}) \dot{s} \geq 0, \quad-\infty<\dot{s}<\infty
$$

The hatched region in Figure 4 comprises the set of all points $(\dot{s}, f)$ with $f_{m} \leq f \leq$ $f_{M}$ for which the entropy inequality $f \dot{s} \geq 0$ holds; the curve described by (3.7), (3.8) must lie in this hatched region. For present purposes, we shall assume that the graph of the function $\varphi$ has the form indicated schematically by the curve $K$ in Figure 4: $\varphi$ is to be monotonically increasing and smooth everywhere except possibly at $\dot{s}=0$. We let $\varphi(0+)=f_{M}^{0} \geq 0, \varphi(0-)=f_{m}^{0} \leq 0$, where $f_{M}^{0}$ and $f_{m}^{0}$ are new material parameters such that $0 \leq f_{M}^{0} \leq f_{M}, f_{m} \leq f_{m}^{0} \leq 0$, and we assume that $\varphi(+\infty)=f_{M}, \varphi(-\infty)=f_{m}$, where $f_{M}$ and $f_{m}$, introduced earlier, are the maximum and minimum values of driving traction in a 1,3-quasi-static motion. When $f_{M}^{0}>0$ and $f_{m}^{0}<0$, so that $\varphi(\dot{s})$ is discontinuous at $\dot{s}=0$, no motion of the phase boundary is possible for values of the driving traction between $f_{m}^{0}$ and $f_{M}^{0}$, and $f_{M}^{0}$, reflecting a frictional type of relationship between driving traction and phase boundary velocity. It may happen that $f_{m}^{0}=f_{M}^{0}=0$, in which case $\varphi(\dot{s})$ is continuous at $\dot{s}=0$, and motion of the phase boundary occurs whenever the driving traction is different from zero.

In view of our monotonicity assumption above, the relation (3.7) can also be represented in inverted form: $\dot{s}=\Phi(f), f_{m} \leq f \leq f_{M}$.

Since the driving traction $f(t)$ is determined through (2.6) by the strains $\frac{ \pm}{\gamma}(t)$ at the particles on either side of the phase boundary, the kinetic relation (3.7) involves only the quantities $\stackrel{ \pm}{\gamma}(t)$ and $\dot{s}(t)$ at the phase boundary and is thus purely local in character. The statement (3.7) is also problem-independent. Moreover, a relation of the form (3.7) can be generalized to three-dimensional thermomechanical processes in thermoelastic materials, as shown in [4]. It is worth noting that the nominally more general kinetic relation

$$
\dot{s}=\Psi(\bar{\gamma}, \stackrel{+}{\gamma})
$$

where $\Psi$ is a constitutive function defined on $\left(-1, \gamma_{m}\right] \times\left[\gamma_{M}, \infty\right)$, is in fact equivalent 
Figure 4. Kinetic relation $f=\varphi(\dot{s})$.

There are several sources of motivation for the form (3.7) of the kinetic relation assumed here. The first of these is to be found in the formalism of internal variable theory. In the description of the macroscopic response of the bar, the force $F$ and the elongation $\delta$ play the role of macroscopic "stress-like" and "strain-like" variables, respectively, while the phase boundary location $s$ is an "internal variable". If $E(F, s)$ is the total strain energy in the bar corresponding to a given force $F$ and a given location $s$ of the phase boundary, and if $U(F, s)=E(F, s)-F \Delta_{13}(F, s)$ is the corresponding total potential energy, then one can show that $\partial U(F, s) / \partial F=-\delta$ and $\partial U(F, s) / \partial s=-f A$. Thus the driving force $f A$ and the internal variable $s$ are conjugate to each other in the same sense that the macroscopic variables $F$ and $\delta$ are conjugate. It is well known that, in internal variable formalisms, one must specify an evolution law for the internal variable; perhaps the simplest and most natural example of such a law in the present setting is one that relates $\dot{s}$ and the 
chanical process. It was shown there that the total rate of entropy production is due to two sources: one, arising from heat conduction and involving the product of heat flux $\mathbf{q}$ and the gradient of the reciprocal of the local temperature $\theta$, represents the contribution to entropy production at points where the thermo-mechanical fields are smooth. The second accounts for the entropy production at a strain discontinuity and is given by the product of $\mathcal{F} / \theta$ and $\dot{s}$, where $\dot{s}$ is the local velocity of the discontinuity, and $\mathcal{F}$ is the thermomechanical counterpart of the driving traction $f$; indeed, $\mathcal{F}$ reduces to $f$ for isothermal processes in a bar. According to the flux-affinity formalism, one would identify the heat flux $\mathbf{q}$ and the velocity $\dot{s}$ as "fluxes", and the gradient of $1 / \theta$ and $\mathcal{F} / \theta$ as respective corresponding "affinities" in the above representation of the entropy production rate. The formalism further calls for constitutive laws relating fluxes and affinities. For the simplest case of "purely resistive materials", these laws relate each affinity to its associated flux. In the setting described above, $\mathbf{q}$ would be a materially-determined function of the gradient of $1 / \theta$, corresponding to a definite heat conduction law, and $\mathcal{F} / \theta$ would be a materially-determined function of $\dot{s}$. For isothermal processes, the latter relationship would correspond precisely to a kinetic relation of the form (3.7). For discussions of the flux-affinity formalism, see Chapter 14 of [8] and Lecture 7 of [42].

The basic principles of continuum theory do not impose any further restrictions on, or provide examples of, kinetic response functions $\varphi$. These must be supplied by appropriate constitutive modeling. For example, the choice $\varphi(\dot{s}) \equiv 0$ for the kinetic response function would result from a model in which all motions of a phase boundary are dissipation-free. For nearly dissipation-free motions of the bar, $\varphi(\dot{s})=$ $\kappa \dot{s}$ with $\kappa$ a sufficiently small positive constant, is natural. At the other extreme is a kinetic response function, to be discussed in Subsection 5.3, that corresponds to a definite notion of maximally dissipative phase boundaries.

As an example of more detailed modeling at the microscopic level, we outline the derivation of a kinetic relation based on the classical notion of "thermally activated" phase transitions. Let the potential $g(\gamma ; \sigma)$ be defined by

$$
g(\gamma ; \sigma)=W(\gamma)-\sigma \gamma, \quad \gamma>-1, \quad \sigma>-\mu_{1}
$$

If $\sigma<\sigma_{m}$, the function $g(\cdot ; \sigma)$ has a single minimum, and it occurs at a strain in the phase- 1 interval $\left(-1, \gamma_{M}\right)$; if $\sigma>\sigma_{M}, g(\cdot ; \sigma)$ again has one minimum, but now it occurs at a strain in the phase- 3 interval $\left(\gamma_{m}, \infty\right)$. On the other hand, when $\sigma_{m}<\sigma<\sigma_{M}, g(\cdot ; \sigma)$ has two local minima separated by a local maximum; one of these minima occurs in the phase- 1 interval, the other in the phase- 3 interval. Figure 5 shows schematic graphs of $g$ in the various cases.

At a local extremum of $g(\cdot ; \sigma), \gamma$ and $\sigma$ are related by $\sigma=\widehat{\sigma}(\gamma)=W^{\prime}(\gamma)$, so that, in an actual equilibrium state, the strain at each particle of the bar must be an 
in front of the interface thus "jumps" from one local minimum to the other. The kinetic relation is now constructed by viewing this jumping process on an atomic scale: atoms undergo random thermal fluctuations in their positions and velocities, and their energies vary accordingly. In order to jump from one minimum of $g$ to the other, an atom must acquire an energy at least as great as that represented by the relevant "energy barrier": for an atom undergoing a $1 \rightarrow 3$ transition by jumping from the minimum on the left to the one on the right in Figure 5 , this barrier is $b_{13}$; for the reverse transition, it is $b_{31}$. Under suitable assumptions about the statistics of this process, the probability that the energy of an atom is at least as great as, say, $b$ is $\exp (-r b)$, where $r$ is a constant. Next, the average rate at which atoms jump from one minimum to the other is taken to be proportional to the probability of exceeding the corresponding energy barrier; we assume for simplicity that the proportionality factor is the same for $1 \rightarrow 3$ and $3 \rightarrow 1$ transitions. The velocity $\dot{s}$ of a 1,3-phase boundary, being a macroscopic measure of the net rate at which atoms change from phase 3 to phase 1 , is then taken to be the difference in the average rates associated with the $1 \rightarrow 3$ and the $3 \rightarrow 1$ transitions:

$$
\dot{s}=R\left(e^{-r b_{31}}-e^{-r b_{13}}\right),
$$

where $R$ is a positive proportionality constant. For the trilinear material of Figure 1, the energy barriers $b_{31}$ and $b_{13}$ at a stress level $\sigma$ are found to be given by

$$
b_{31}=\frac{1}{2}\left(\frac{1}{\mu_{3}}-\frac{1}{\mu_{1}}\right) \frac{\sigma_{M}}{\sigma_{M}-\sigma_{m}}\left(\sigma-\sigma_{m}\right)^{2}, b_{13}=\frac{1}{2}\left(\frac{1}{\mu_{3}}-\frac{1}{\mu_{1}}\right) \frac{\sigma_{m}}{\sigma_{M}-\sigma_{m}}\left(\sigma-\sigma_{M}\right)^{2} .
$$

From (3.11), (3.12), one has

$$
\dot{\boldsymbol{s}}=R\left[e^{-\lambda \sigma_{M}\left(\sigma-\sigma_{m}\right)^{2}}-e^{-\lambda \sigma_{m}\left(\sigma-\sigma_{M}\right)^{2}}\right],
$$

where

$$
\lambda=\frac{r}{2}\left(\frac{1}{\mu_{3}}-\frac{1}{\mu_{1}}\right) \frac{1}{\sigma_{M}-\sigma_{m}} .
$$

From (3.5), one can express the stress $\sigma$ in terms of the static driving traction $f$ as

$$
\sigma=\sigma_{0}\left(1-f / f_{0}\right)^{1 / 2}
$$

where $\sigma_{0}=\left(\sigma_{M} \sigma_{m}\right)^{1 / 2}$ is the Maxwell stress, and $f_{0}=(1 / 2)\left(\mu_{1}-\mu_{3}\right) \gamma_{M} \gamma_{m}$. The final form of the thermal activation-based kinetic relation then follows from (3.13) and (3.15):

$$
\dot{s}=\Phi(f) \equiv R\left\{e^{-\lambda \sigma_{M}\left[\sigma_{0}\left(1-f / f_{0}\right)^{1 / 2}-\sigma_{m}\right]^{2}}-e^{-\lambda \sigma_{m}\left[\sigma_{0}\left(1-f / f_{0}\right)^{1 / 2}-\sigma_{M}\right]^{2}}\right\}
$$


lies in a certain finite interval. Finally, it should be pointed out that the statistical parameters $r$ and $R$ entering (3.16), and for that matter the mechanical parameters as well, are dependent upon the absolute temperature $\theta$; indeed, $r=1 /(k \theta)$, where $k$ is proportional to Boltzmann's constant.

Kinetic relations not of the form (3.7) may sometimes be of interest. One that is suggested by adapting to the present one-dimensional context a dislocationbased theory put forward by Grujicic, Olson and Owen [17] for the modeling of fast martensitic phase transitions is $f=f_{*}+m \ddot{s}+\kappa \dot{s}$, with $f_{*}, m$ and $\kappa$ constants.

Figure 5. The potential $g(\gamma ; \sigma)$ with the energy barriers $b_{13}$ and $b_{31}$.

3.3. A nucleation condition. Once a 1,3-phase boundary has been initiated, the kinetic relation (3.7) controls its evolution. A separate nucleation criterion is needed to signal the emergence of such a phase boundary from a single-phase configuration. In general, a particle may change its phase in one of two ways: a preexisting phase boundary may pass through it, or it may undergo a spontaneous phase 
We further assume that the occurrence of such a spontaneous $1 \rightarrow 3$ transition from a uniform state is controlled by a critical value, say $f_{13}$, of driving traction $f$ : if the stress $\sigma$ at the particle labeled by $x=L$ has increased to a value such that, according to (3.5), the corresponding driving traction $f$ coincides with $f_{13}$, this particle will spontaneously transform to phase 3 , thus initiating a 1,3-phase boundary at $x=L$. This phase boundary then moves leftward into the bar; its initial velocity will be determined by (3.7) with $f=f_{13}$. By (3.5), the stress $\sigma=\sigma_{13}$ corresponding to the nucleation driving traction $f=f_{13}$ is

$$
\sigma_{13}=\sigma_{0}\left(1-f_{13} / f_{0}\right)^{1 / 2}
$$

Since by entropy inequality $f_{13} \leq 0, \sigma_{13}$ is at least as great as the Maxwell stress.

Similarly, if the bar is in a pure phase- 3 state, spontaneous transformation from phase 3 to phase 1 of the particle labeled by $x=0$ is assumed to occur when the stress $\sigma$ has diminished to a value such that the associated driving traction in (3.5) achieves a critical level $f_{31} \geq 0$; the corresponding critical stress in this case is less than $\sigma_{0}$. Under these circumstances, a 1,3-phase boundary is initiated at $x=0$, and it moves with a positive initial velocity determined by (3.7) with $f=f_{31}$. The initiation levels of driving traction $f_{13}$ and $f_{31}$ are viewed as material parameters that satisfy the following inequalities: $f_{m}<f_{13} \leq f_{m}^{0} \leq 0 \leq f_{M}^{0} \leq f_{31}<f_{M}$; see Figure 4.

As shown in detail in [2], a kinetic relation (3.7) in which $\varphi$ has the form shown in Figure 4, together with the nucleation criterion described above, gives rise to a fully determinate quasi-static macroscopic response. When the bar is first loaded from an unstressed reference state and then unloaded back to that reference state, the associated force-elongation curve is generally hysteretic and rate-dependent. An example of the force-elongation response corresponding to such a loading-unloading program is shown schematically in Figure 6. For a suitable choice of the kinetic response function $\varphi$ and the nucleation values $f_{13}, f_{31}$ of driving traction, the calculated macroscopic response is qualitatively similar to that observed in tension-bar experiments involving slowly propagating phase boundaries for martensitic transformations in certain alloys; such experiments have been reported, for example, by Grujicic, Olson and Owen [18] and by Krishnan and Brown [25]. 
Figure 6. Force-elongation curve for loading followed by unloading.

4. The dynamical theory. The need for additional constitutive information can be seen in the dynamical theory of phase transformation as well as in quasistatics. In the present section, we describe how this issue can be illuminated by studying the Riemann problem for the dynamical field equations and jump conditions (2.1)-(2.4). We begin by reviewing the local structure of discontinuities when inertial effects are accounted for. We then describe our results for the Riemann problem for the trilinear material, which make it clear that uniqueness fails, even when the entropy inequality $(2.8),(2.6)$ is in force. Finally, we show how the nucleation criterion and the kinetic relation, appropriately adapted to dynamics, can be used to remedy the constitutive deficiency.

4.1. Local structure of discontinuities. At a moving discontinuity $x=s(t)$, the jump conditions (2.3), (2.4) imply

$$
\rho \dot{s}^{2}=\frac{\widehat{\sigma}(\grave{\gamma})-\widehat{\sigma}(\bar{\gamma})}{+}
$$


that the right side of (4.1) is nonnegative, then it is possible to find numbers $\bar{v}, \stackrel{+}{v}$ and $\dot{s}$ such that the pair of jump conditions (2.3), (2.4) are satisfied.

The requirement (4.1) rules out shock waves of 2,2-type. In addition, for the trilinear material of Figure 1, it shows that shock waves of 1,1-type and 3,3-type have constant propagation speeds $\pm c_{1}$ and $\pm c_{3}$, respectively, where

$$
c_{1}=\left(\mu_{1} / \rho\right)^{1 / 2}, \quad c_{3}=\left(\mu_{3} / \rho\right)^{1 / 2}<c_{1} .
$$

At a shock wave of either 1,1- or 3,3-type, the linearity of the stress response function $\widehat{\sigma}(\gamma)$ between the strains $\bar{\gamma}$ and $\stackrel{+}{\gamma}$ allows one to infer from (2.6) that the driving traction vanishes, so that the entropy inequality (2.8) is automatically satisfied at such a shock wave.

Turning next to phase boundaries, we suppose that $\bar{\gamma}$ and $\stackrel{+}{\gamma}$ belong to different phases. Since we shall not encounter cases in which either $\bar{\gamma}$ or $\stackrel{+}{\gamma}$ is in phase 2 (the "unstable phase"), we may without loss of generality take $\bar{\gamma}$ to be in phase 1 and $\hat{\gamma}$ in phase 3 , so that the associated discontinuity at $x=s(t)$ is a 1,3 -phase boundary. When specialized to this case and to the trilinear material, (4.1) becomes

$$
\dot{s}^{2}=\frac{c_{3}^{2} \stackrel{+}{\gamma}-c_{1}^{2} \bar{\gamma}}{\stackrel{+}{\gamma}-\bar{\gamma}}
$$

In the $\bar{\gamma}, \stackrel{+}{\gamma}$-plane, the set of pairs $(\bar{\gamma}, \stackrel{+}{\gamma})$ for which $\bar{\gamma}$ is in phase $1, \stackrel{+}{\gamma}$ is in phase 3 and the right-side of (4.4) is non-negative is represented by the region $\Gamma$ shown partly hatched and partly shaded in Figure 7. Any any point on the boundary segment BC, the numerator in (4.4) vanishes, so that $\dot{s}=0$; the corresponding phase boundary is thus instantaneously stationary. The point $M$ in the figure corresponds to the so-called Maxwell state, which is the special equilibrium phase mixture for which $\bar{\gamma}=\sigma_{0} / \mu_{1}, \stackrel{+}{\gamma}=\sigma_{0} / \mu_{3}$, where $\sigma_{0}$ is the Maxwell equal-area stress.

The driving traction $f$ acting on a phase boundary of 1,3-type can be found from (2.6) and the explicit form of $\widehat{\sigma}(\gamma)$ for the trilinear material:

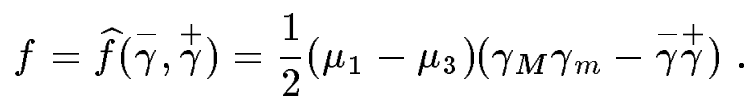

(Equation (4.5) yields the formula (3.5) for the static driving traction in the equilibrium case, for which $\bar{\sigma}=\mu_{1} \bar{\gamma}=\stackrel{+}{\sigma}=\mu_{3} \stackrel{+}{\gamma}$.) It follows from (4.5) that the driving traction vanishes on the hyperbola $\bar{\gamma} \hat{\gamma}=\gamma_{M} \gamma_{m}$ in the $\bar{\gamma}, \stackrel{+}{\gamma}$-plane. Thus according to $(2.5)$, points in $\Gamma$ that lie on this hyperbola correspond to values of $\bar{\gamma}$ and $\stackrel{+}{\gamma}$ for which the associated phase boundary $x=s(t)$ propagates without dissipation. 
Figure 7. The region $\Gamma$ in the $\bar{\gamma}, \stackrel{+}{\gamma}$-plane.

With the help of (4.4) and (2.8), one can show as in [5] that the velocity $\dot{s}$ of a 1,3 -phase boundary necessarily lies in the range

$$
-c_{3}<\dot{s}<c_{*},
$$

where $\rho c_{*}^{2}$ is the slope of the chord joining the point $(-1, \widehat{\sigma}(-1))$ to $\left(\gamma_{m}, \sigma_{m}\right)$ in the $\gamma, \sigma$-plane of Figure 1: $c_{*}=\left\{\left(c_{1}^{2}+\gamma_{m} c_{3}^{2}\right) /\left(1+\gamma_{m}\right)\right\}^{1 / 2}, c_{3}<c_{*}<c_{1}$. The propagation speed $|\dot{s}|$ is said to be subsonic if $|\dot{s}|<c_{3}$. By (4.4), this occurs only 
the entropy inequality (2.8). If $f=0$, the point $(\bar{\gamma}, \stackrel{+}{\gamma})$ lies on the hyperbola; such a point maps to a pair of points $( \pm \dot{s}, 0)$ in the $\dot{s}, f$-plane that satisfy the entropy inequality trivially and for which $0 \leq \dot{s} \leq c_{3}$. All points on $B C$ map to points on the $f$-axis; again the entropy inequality is trivially satisfied. All points in $\Gamma$ that lie on $\bar{\gamma}=0$ map to the same pair of points $\left( \pm c_{3}, f_{0}\right)$, where $f_{0}=(1 / 2)\left(\mu_{1}-\mu_{3}\right) \gamma_{m} \gamma_{M}$. The union of the hatched and shaded regions shown in Figure 8 represents the set of those points in the $\dot{s}, f$-plane that come from points in $\Gamma$ and are consistent with the entropy inequality. The upper and lower boundary curves of the hatched region are described by

$$
f=f_{M}(\dot{s}) \equiv\left\{\begin{array}{l}
0, \quad-c_{3}<\dot{s}<0, \\
f_{0}\left(1-\frac{\gamma_{m}}{\gamma_{M}} \frac{c_{3}^{2}-\dot{s}^{2}}{c_{1}^{2}-\dot{s}^{2}}\right), 0 \leq \dot{s}<c_{3},
\end{array} f=f_{m}(\dot{s}) \equiv\left\{\begin{array}{l}
f_{0}\left(1-\frac{\gamma_{M}}{\gamma_{m}} \frac{c_{1}^{2}-\dot{s}^{2}}{-c_{3}^{2}-\dot{s}^{2}}\right),-c_{3}<\dot{s} \leq 0 \\
0, \quad 0<\dot{s}<c_{3} .
\end{array}\right.\right.
$$


4.2. The Riemann problem. In this problem, one seeks solutions on the upper half of the $x, t$-plane of the field equations (2.1), (2.2) and the jump conditions $(2.3),(2.4)$ that satisfy the entropy inequality (2.8) as well as the initial conditions

$$
\gamma(x, 0+)=\left\{\begin{array}{ll}
\gamma_{L}, & -\infty<x<0, \\
\gamma_{R}, & 0<x<+\infty,
\end{array} \quad v(x, 0+)= \begin{cases}v_{L}, & -\infty<x<0, \\
v_{R}, & 0<x<+\infty .\end{cases}\right.
$$

Here $\gamma_{L}, \gamma_{R}, v_{L}$ and $v_{R}$ are given constants. We seek solutions that are piecewise smooth and invariant under the scale change $x \rightarrow k x, t \rightarrow k t$, where $k$ is any constant. We restrict attention to the case in which neither of the initial strains $\gamma_{L}$ nor $\gamma_{R}$ belongs to phase 2 (the unstable phase), but we do admit the possibility that at a subsequent time $t>0$, the strain field might involve this phase.

Solutions of the Riemann problem that are scale-invariant must be constant in various sectors of the $x, t$-plane:

$$
\gamma(x, t)=\gamma_{j}, \quad v(x, t)=v_{j}, \quad \dot{s}_{j} t<x \leq \dot{s}_{j+1} t, \quad j=0,1, \ldots, N
$$

where $\gamma_{j}, v_{j}, \dot{s}_{j}$ and $N$ are constants, with $N$ a non-negative integer, and $\gamma_{0}=$ $\gamma_{L}, \gamma_{N}=\gamma_{R}, v_{0}=v_{L}, v_{N}=v_{R}, \dot{s}_{0}=-\infty, \dot{s}_{N+1}=+\infty$. The case $N=0$ can occur only if $\gamma_{L}=\gamma_{R}, v_{L}=v_{R}$. The $\gamma_{j}$ 's must satisfy $\gamma_{j}>-1$ for $j=0, \ldots, N$; if $N>0$, one must also have $\gamma_{j} \neq \gamma_{j+1}$ for $j=0, \ldots, N-1$. In the space-time field given by (4.9), there are $N$ strain discontinuities on lines $x=\dot{s}_{j} t$; they may be shock waves or phase boundaries. For the trilinear material, fans cannot occur. If $x=\dot{s}_{j} t$ is a shock wave, then $\dot{s}_{j}$ must take one of the four values $\pm c_{1}, \pm c_{3}$. At each discontinuity, the jump conditions $(2.3),(2.4)$ and the entropy inequality (2.8) are required to hold.

The nature of the solutions to the Riemann problem posed above depends on the phases of the initial strains $\gamma_{L}, \gamma_{R}$. Before describing the solutions, it is convenient to introduce a parameter $h$ that depends only on the given data:

$$
h=\left(c_{L} \gamma_{L}+c_{R} \gamma_{R}+v_{R}-v_{L}\right) /\left(c_{L}+c_{R}\right) .
$$

Here $c_{L}$ and $c_{R}$ are the speeds of shock waves associated with the phases of the given strains $\gamma_{L}$ and $\gamma_{R}$; thus, for example, $c_{L}=c_{1}$ if $\gamma_{L}$ is in phase $1, c_{L}=c_{3}$ if $\gamma_{L}$ is in phase 3 .

A derivation of the results that are stated without proof below can be found in $[5]$.

Case 1. Initial strains in distinct phases. In this case, we may assume without loss of generality that $\gamma_{L}$ is in phase $1, \gamma_{R}$ in phase 3 . The entropy inequality $(2.8)$ 
data when $h>0$, so that there is in fact a one-parameter family of solutions, each satisfying the entropy inequality, to the Riemann problem for given initial data in this sub-case. In contrast, when the initial data are such that $h<0$, there is no solution satisfying the entropy inequality unless $h$ lies in a definite range; when a solution exists, it is unique, and it is of the form illustrated in Figure 9(b), with one shock wave and a supersonic phase boundary. Thus when the initial data are in distinct phases, the Riemann problem for the trilinear material reveals a constitutive deficiency for subsonically propagating phase boundaries in that the velocity of the interface fails to be determined by the data. In contrast, supersonically propagating phase boundaries require no further information for their full determination from initial data. This appears to be reminiscent of the distinction between deflagrations and detonations in combustion theory; see p. 225 of the book by Courant and Friedrichs [11] and Section D,3 of the monograph by Hayes [21]. 
Case 2. Initial strains in a common phase. Next consider the case in which the initial strains are both in the same phase: thus suppose, for example, that $\gamma_{L}$ and $\gamma_{R}$ are both in phase 1 . The entropy inequality can be invoked to show that any solution of the Riemann problem in this case must necessarily take one of the two forms shown in Figure 10; again, phase 2 is absent. When the initial data are such that $h>\sigma_{0} / \mu_{1}$, where $\sigma_{0}$ is the Maxwell stress (Figure 1), there is a twoparameter family of solutions satisfying the entropy inequality of the form shown in Figure 10(b); the two parameters are the velocities $\dot{s}$ and $\dot{\boldsymbol{s}}_{*}$ of the two subsonically propagating phase boundaries. On the other hand, when $-1<h \leq \sigma_{M} / \mu_{1}$, there is a solution of the form shown in Figure 10(a) satisfying the entropy inequality at all discontinuities and involving no phase boundaries. In any solution of the two-parameter family arising in the first subcase, the bar ultimately changes phase (from 1 to 3 ), whereas in the second subcase, the bar remains for all time in phase 1. 
Supersonic phase boundaries cannot arise in this case; they can occur when the initial data involve strains $\gamma_{L}$ and $\gamma_{R}$ that are both in phase 3 ; in this situation, they appear only in solutions that are uniquely determined by the initial data, as in Case 1.

The foregoing discussion of the Riemann problem reveals two distinct types of non-uniqueness. First, there is the lack of uniqueness arising because of the undetermined velocities of subsonically moving phase boundaries in both Cases 1 and 2. Second, in Case 2, for initial data such that $\sigma_{0} / \mu_{1}<h \leq \sigma_{M} / \mu_{1}$, two types of solutions can occur: one in which the bar ultimately changes phase, the other in which it does not. Moreover, we also learn from the results summarized above that no lack of uniqueness of solutions satisfying the entropy inequality occurs in connection with supersonically propagating phase boundaries.

4.3. Kinetics, nucleation and uniqueness. We now describe how the nucleation criterion and the kinetic relation (3.7), when suitably adapted to dynamics, are sufficient to remedy the lack of uniqueness described above.

In the quasi-static theory, it was required that the curve in the $\dot{s}, f$-plane represented by the kinetic relation $f=\varphi(\dot{s})$ should lie in the hatched region of Figure 4 . In the dynamical setting, this requirement must be modified in two ways. First, the set of dynamically permissible pairs $(\dot{s}, f)$ consists of the union of the hatched and shaded regions in Figure 8 , which clearly differs from its quasi-static counterpart in Figure 4. Second, the discussion of the Riemann problem makes it clear that a kinetic relation cannot be accommodated by supersonically moving phase boundaries, so that the curve in the $\dot{s}, f$-plane represented by the kinetic relation must be confined to the hatched region in Figure 8. Thus we replace (3.7) with

$$
f=\varphi(\dot{s}),-c_{3}<\dot{s}<c_{3},
$$

retaining the requirement (3.8) imposed by the entropy inequality, but with $\dot{s}$ restricted to the subsonic regime. The curve $K^{\prime}$ represented by (4.11) is shown schematically in Figure 8.

As in the quasi-static theory, the apparently more general form (3.9) of the kinetic relation is in fact equivalent to a relation between $\dot{s}$ and $f$ in the dynamic case as well, since there is a one-to-one relationship between pairs $(\bar{\gamma}, \stackrel{+}{\gamma})$ and pairs $(\dot{s}, f)$.

Let us now consider Case 1 of the Riemann problem. When the initial data are such that $h>0$, it is possible to show that the kinetic relation (4.11), with $\varphi$ monotonically increasing, serves to determine the subsonic phase boundary velocity $\dot{s}$, so the the Riemann problem, when augmented by the kinetic relation, has a unique solution. Since two phases are present initially, the nucleation criterion plays 
there is a two-parameter family of solutions (Figure 10(b)); each solution in this family involves two phase boundaries and leads to a change of phase of the bar. Finally, for each $h$ in the intermediate interval $\left[\sigma_{0} / \mu_{1}, \sigma_{M} / \mu_{1}\right]$, solutions of both of these types are available. Within the class of solutions for which the bar changes phase, uniqueness is secured by imposing the appropriate kinetic relation at each of the two phase boundaries, thereby determining their respective velocities. The only remaining uniqueness issue arises for values of $h$ in the intermediate range $\left[\sigma_{0} / \mu_{1}, \sigma_{M} / \mu_{1}\right]$; this this subcase, it is required to choose between precisely two solutions, in one of which the bar changes phase, while in the other it does not. In order to make this choice, one appeals to the nucleation criterion, according to which a phase transition is initiated at a critical level of driving traction. As shown in [5], this leads to the conclusion that the change-of-phase solution is selected when $\sigma_{13} / \mu_{1} \leq h<\sigma_{M} / \mu_{1}$, with $\sigma_{13}$ given by (3.17). For $\sigma_{0} / \mu_{1}<h<\sigma_{13} / \mu_{1}$, the criterion picks the solution with no phase change. Thus after imposing both the nucleation criterion and the kinetic relation, all uniqueness issues in Case 2 of the Riemann problem are resolved.

5. Admissibility criteria. For an elastic material with a monotonically rising stress-strain curve whose curvature does not change sign, the entropy inequality (2.8) selects a unique solution from the multiplicity of solutions to the Riemann problem that would otherwise exist; the role of the entropy inequality in this respect is familiar from gas dynamics. However, when the stress-strain curve is neither strictly convex nor strictly concave, the entropy inequality is not strong enough to single out solutions. Most approaches that have been designed to confront this lack of uniqueness involve replacing the entropy inequality by a stronger selection mechanism, or "admissibility criterion". A number of such criteria have been studied in the literature; here we briefly discuss three of them; the viscosity-strain-gradient criterion, the entropy rate shock criterion and the chord criterion. In each case, at a phase boundary, the admissibility criterion turns out to be equivalent to a particular kinetic law that relates the driving traction $f$ to the phase boundary velocity $\dot{s}$.

5.1. The viscosity-strain gradient admissibility criterion. In order to study the admissibility of a phase boundary according to this criterion, one replaces the constitutive statement $\sigma=\widehat{\sigma}(\gamma)$ appropriate to the elastic bar by one that adds contributions due to viscosity and strain gradient to the elastic part of the stress. Thus one takes

$$
\sigma=\widehat{\sigma}(\gamma)+\rho \nu \gamma_{t}-\rho \lambda \gamma_{x x}
$$

where $\nu \geq 0$ and $\lambda \geq 0$ are the viscosity and the strain-gradient coefficient, respectively; the dimensionless parameter $\omega=2 \lambda^{1 / 2} / \nu$ will also be convenient. The 
When considering the material governed by (5.1), one strengthens the smoothness requirements on the displacement field: $u$ is now to be twice continuously differentiable with piecewise continuous third and fourth derivatives in all parts of space-time to be considered. Of the jump conditions $(2.3),(2.4)$, the latter is then trivially satisfied, and the former reduces to the requirement that $\sigma$ be continuous; by (5.1), this requires that $\gamma_{x x}$, and therefore $u_{x x x}$, be continuous.

A phase boundary in the elastic bar is said to be admissible according to the viscosity-strain gradient criterion if the states $(\bar{\gamma}, \bar{v}),(\stackrel{+}{\gamma}, \stackrel{+}{v})$ on either side of the interface can be smoothly connected by a traveling wave in the augmented theory, i.e. if there is a solution of the form

$$
\gamma=\gamma(\xi), \quad v=v(\xi), \quad \xi=x-\dot{s} t
$$

of equations (2.1), (2.2) and (5.1), where $\dot{s}$ is a constant, and

$$
\gamma(-\infty)=\bar{\gamma}, \quad v(-\infty)=\bar{v}, \quad \gamma(+\infty)=\stackrel{+}{\gamma}, \quad v(+\infty)=\stackrel{+}{v}
$$

Our interest here is in 1,3-phase boundaries, so we shall take $\bar{\gamma}$ in phase $1, \stackrel{+}{\gamma}$ in phase 3 .

One can eliminate the velocity field $v(x, t)$ from the discussion and state this admissibility criterion in terms of strains alone: a 1,3-phase boundary in the elastic material is said to be admissible according to the viscosity-strain gradient criterion if there exists a solution $\gamma(\xi)$, twice continuously differentiable on $(-\infty, \infty)$, of the following travelling wave problem:

$$
\begin{gathered}
\lambda \gamma^{\prime \prime}+\nu \dot{s} \gamma^{\prime}-\frac{\widehat{\sigma}(\gamma)}{\rho}+\dot{s}^{2} \gamma=-\frac{\widehat{\sigma}(\bar{\gamma})}{\rho}+\dot{s}^{2} \bar{\gamma} \quad \text { on } \quad(-\infty, \infty) \\
\gamma(-\infty)=\bar{\gamma}, \quad \gamma(+\infty)=\dot{\gamma}
\end{gathered}
$$

If the travelling wave problem has a solution, then one can show directly from (5.4), (5.5) that the given strains $\bar{\gamma}, \stackrel{+}{\gamma}$ must necessarily be such that

$$
\frac{\widehat{\sigma}(\stackrel{+}{\gamma})-\widehat{\sigma}(\bar{\gamma})}{\dot{\gamma}-\bar{\gamma}} \geq 0
$$

and that $\dot{s}^{2}$ is then given by (4.1); moreover, because $\nu$ is non-negative, the sign of $\dot{s}$ must necessarily be consistent with the entropy $f \dot{s} \geq 0$, where $f$ is expressed in 
have a solution. In contrast, it is also shown in [6] that, if the propagation speed is subsonic, the travelling wave problem has a solution if and only if (5.6) and the following relation both hold:

$$
f=\varphi(\dot{s}) \equiv \frac{1}{2}\left(\mu_{1}-\mu_{3}\right)\left[\gamma_{M} \gamma_{m}-\frac{G^{2}(\dot{s}, \omega)}{\left(c_{1}^{2}-\dot{s}^{2}\right)\left(c_{3}^{2}-\dot{s}^{2}\right)}\right], \quad-c_{* *}<\dot{s}<c_{* *}
$$

Here $f$ is again given in terms of the data $\bar{\gamma}, \stackrel{+}{\gamma}$ by $(2.6)$, the parameter $c_{* *}$ limiting the range of $\dot{s}$ is defined by

$$
c_{* *}=\left\{\begin{array}{cl}
{\left[\omega^{2} /\left(1-\omega^{2}\right)\right]^{1 / 2} c_{2} \leq c_{3}} & \text { if } 0<\omega^{2} \leq c_{3}^{2} /\left(c_{2}^{2}+c_{3}^{2}\right) \\
c_{3} & \text { if } \omega^{2} \geq c_{3}^{2} /\left(c_{2}^{2}+c_{3}^{2}\right),
\end{array}\right.
$$

where $\omega$ is defined below (5.1), and $c_{2}=\left(\mu_{2} / \rho\right)^{1 / 2}$ (see Figure 1 for the meaning of $\left.\mu_{2}\right)$. The function $G(\cdot, \omega)$ in $(5.7)$, whose representation is too complicated to display here, is fully and explicitly determined in [6]. For present purposes, it is sufficient to note that, for each $\omega, G(\cdot, \omega)$ is continuous on $\left[-c_{* *}, c_{* *}\right]$ and has a first-order zero at $\dot{s}=c_{3}$ when $c_{* *}=c_{3}$.

One can show that the solution $\gamma(\xi)$ of the traveling wave problem has a limit as the viscosity $\nu$ tends to zero with the parameter $\omega$ and the data $\bar{\gamma}, \stackrel{+}{\gamma}$ held fixed. Indeed, $\gamma(\xi)$ tends to $\bar{\gamma}$ for every $\xi<0$ and to $\stackrel{+}{\gamma}$ for every $\xi>0$ in this limit. The zero-viscosity limit of $\gamma(\xi)$ satisfies all the differential equations and jump conditions of the elastic theory. Within that theory, it represents a propagating phase boundary moving with a velocity $\dot{s}$ that satisfies the jump condition (4.1), the entropy inequality $(2.8),(2.6)$, and the relation $(5.7)$, which is unaffected by the limit process because $G(\dot{s}, \omega)$ depends on $\nu$ and $\lambda$ only through $\omega$. In the framework of the elastic theory, (5.7) represents a kinetic relation of the form (3.7) inherited from the admissibility criterion associated with the augmentation (5.1).

Figure 11 describes this particular kinetic relation by plotting $f / f_{0}$ vs. $\dot{s} / c_{3}$ according to (5.7); here $f_{0}=\left(\mu_{1}-\mu_{3}\right) \gamma_{M} \gamma_{m} / 2$. One finds that $f / f_{0}$ depends only on the three material parameters $\sigma_{M} / \sigma_{m}, \gamma_{M} / \gamma_{m}$ and $\omega$; the figure is plotted for fixed values of the first two of these and for various values of $\omega$. For small values of $\omega$ (corresponding, for example, to large viscosity), the graph strikes the top and bottom boundaries of the permissible region in the $\dot{s}, f$-plane when $\dot{s}=c_{* *}$. This occurs when $c_{* *}<c_{3}$, i.e. when $\omega<c_{3} /\left(c_{2}^{2}+c_{3}^{2}\right)^{1 / 2}$. For the other values of $\omega$ considered, $f / f_{0}$ approaches $-\infty$ as $\dot{s} / c_{3}$ tends to -1 , but $f / f_{0}$ tends to unity as the latter ratio approaches +1 . For large values of $\omega$, or small viscosity, the kinetic relation corresponds to nearly dissipation-free motion of the phase boundary over most of the range of velocity $\dot{s}$. Note from the figure that $\varphi(\dot{s})$ increases monotonically with $\dot{s}$ for the values of the parameters considered. It can be shown 
Figure 11. The kinetic relation (5.7) for $\sigma_{M} / \sigma_{m}=7 / 4, \gamma_{M} / \gamma_{m}=1 / 2$ and various values of $\omega$.

5.2. Entropy-rate shock admissibility criterion. The entropy-rate shock admissibility criterion, which was proposed by Dafermos [12] and studied in connection with phase transitions by Hattori [19,20], James [22] and Pence [31], implements a certain notion of maximum dissipation. A motion of the elastic bar is admissible according to this criterion if each of its propagating strain discontinuities fulfills the following condition at each time instant $t_{*}$ : let $\stackrel{+}{\gamma}\left(t_{*}\right), \stackrel{+}{v}\left(t_{*}\right), \bar{\gamma}\left(t_{*}\right)$ and $\bar{v}\left(t_{*}\right)$ be the instantaneous strains and particle velocities at a discontinuity, and let $D_{*}$ be the 
the competitors, we speak of the modified entropy rate shock admissibility criterion.

Figure 12. Kinetic relation $E$ corresponding to the modified entropy rate shock admissibility criterion for $\sigma_{M} / \sigma_{m}=3 / 2, \gamma_{M} / \gamma_{m}=1 / 5$.

The implications of the modified entropy-rate shock criterion for 1,3-phase boundaries propagating in a trilinear material were explored in [7] by finding explicitly all solutions to the pertinent Riemann problem that satisfy the entropy inequality, and then, for fixed initial data, determining the solution with the greatest dissipation rate. Here we simply describe the relevant results from that paper, to which the reader is referred for further details. It was shown that, while all supersonic 1,3-phase boundaries are found to be admissible according to this criterion, the only subsonic 1,3-phase boundaries that are admissible in the trilinear elastic material are those that satisfy a relation of the form (3.7), with a function $\varphi$ that is explicitly determined in [7] but is too complicated to exhibit here. Thus, for subsonically propagating phase boundaries in the trilinear material, the selection 
monotonically with $\dot{s}$ and satisfies the requirement (3.8). Over two portions of the range of the velocity $\dot{s}$, the curve in the figure coincides with the top and bottom boundaries of the subsonic region in the $\dot{s}, f$-plane in which all kinetic response curves must lie; see also Figure 8.

5.3. Chord admissibility criterion. Finally we turn to the chord criterion for admissibility in the form stated by Shearer [36] for any stress-strain relation. Consider a discontinuity moving with velocity $\dot{s}$ and bearing strains and particle velocities $(\bar{\gamma}, \bar{v}),(\stackrel{+}{\gamma}, \stackrel{+}{v})$ that satisfy the jump conditions $(4.1),(4.2)$. Suppose first that $\bar{\gamma}<\stackrel{+}{\gamma}$; the discontinuity is then said to be admissible according to the chord criterion if one of the following three conditions is satisfied: either $\dot{s}=0$, so that the discontinuity is at rest, or $\dot{s}>0$ and the chord connecting the points $(\bar{\gamma}, \widehat{\sigma}(\bar{\gamma}))$ and $(\stackrel{+}{\gamma}, \widehat{\sigma}(\stackrel{+}{\gamma}))$ on the stress-strain curve lies on or below the curve itself, or $\dot{s}<0$ and the chord lies on or above the stress-strain curve. For admissibility when $\bar{\gamma}>\dot{\gamma}$, the criterion is the same, except that the relative positions of chord and curve as stated above must be reversed. In either case, admissibility according to this criterion requires that either

$$
\dot{s}=0,
$$

or

$$
\dot{s}>0 \text { and } \frac{\widehat{\sigma}(\gamma)-\widehat{\sigma}(\bar{\gamma})}{\gamma-\bar{\gamma}} \geq \frac{\widehat{\sigma}(\hat{\gamma})-\widehat{\sigma}(\bar{\gamma})}{\stackrel{+}{\gamma}-\bar{\gamma}} \text { for all } \gamma \text { between } \bar{\gamma} \text { and } \dot{\gamma}
$$

or

$$
\dot{s}<0 \quad \text { and } \quad \frac{\widehat{\sigma}(\gamma)-\widehat{\sigma}(\bar{\gamma})}{\gamma-\bar{\gamma}} \leq \frac{\widehat{\sigma}(\stackrel{+}{\gamma})-\widehat{\sigma}(\bar{\gamma})}{\stackrel{+}{\gamma}-\bar{\gamma}} \text { for all } \gamma \text { between } \bar{\gamma} \text { and } \dot{\gamma}
$$

A chord criterion similar to the present one was introduced for a single scalar first-order conservation law by Oleinik [29]. Pego [30] has shown that, under suitable assumptions, the chord condition (5.9)-(5.11) is equivalent to the viscosity criterion obtained by setting the strain-gradient coefficient $\lambda$ equal to zero in the viscositystrain gradient criterion discussed in Subsection 5.1.

By making use of the geometric interpretation of the definition (2.6) of driving traction $f$, one finds that the entropy inequality (2.8) is satisfied at any discontinuity admitted by the chord criterion.

For 1,3-phase boundaries in the trilinear material, it is readily seen geometrically that the three alternatives $(5.9)-(5.11)$ are equivalent to the following four alternatives: either 
or

$$
0<\dot{s}<c_{3} \quad \text { and } \quad \stackrel{+}{\gamma}=\gamma_{m}
$$

or

$$
|\dot{s}|>c_{3}
$$

The fourth alternative (5.15) corresponds to supersonic phase boundaries, all of which are admitted by the chord criterion. The remaining possibilities all involve subsonic interfaces. In (5.12), the phase boundary is at rest. When $\dot{s}<0$, the 1,3-phase boundary is advancing into material that is in phase 1 , so at the interface, particles are transforming from phase 1 (the "parent" phase) to phase 3 (the "product" phase); (5.13) states that parent-phase particles that are on the verge of undergoing this $1 \rightarrow 3$ transition always bear the strain $\bar{\gamma}(t)=\gamma_{M}$. Similarly, when the 1,3-phase boundary moves to the right, particles jump from phase 3 to phase 1 , and (5.14) requires that a particle in the parent phase that is about to transform must be at the strain $\gamma_{m}$. Thus with the chord criterion in force, jumping from phase 1 to phase 3 can occur only at the local maximum in the stress-strain curve of Figure 1, while the reverse transition only takes place at the local minimum. This rule for phase transitions corresponds to what is sometimes called the "convention of perfect delay"; see, for example, [32].

Taken together, the restrictions (5.12)-(5.14) imposed by the chord criterion on subsonic 1,3-phase boundaries comprise a kinetic relation connecting driving traction $f$ and phase boundary velocity $\dot{s}$. Indeed, from (4.5), these restrictions are equivalent to

$$
\left.\begin{array}{ll}
f=f_{m}(\dot{s}) & \text { if }-c_{3}<\dot{s}<0, \\
f_{m}(0) \leq f \leq f_{M}(0) & \text { if } \dot{s}=0, \\
f=f_{M}(\dot{s}) & \text { if } 0<\dot{s}<c_{3},
\end{array}\right\}
$$

where $f_{m}(\dot{s})$ and $f_{M}(\dot{s})$, defined in $(4.7)$, correspond respectively to the top and bottom boundaries of the hatched region in Figure 8 . The relation (5.16) can be expressed in the form $\dot{s}=\Phi(f)$. A graph of the kinetic relation (5.16) is shown as the curve $C$ in Figure 13. The curve is reminiscent in some respects of rigid-plastic response with work-hardening or of the force-velocity relationship associated with friction.

The chord condition is equivalent to the kinetic relation $(5.16)$; both in turn are 
Thus the local dissipation rate $D=f \dot{s}$ at a maximally dissipative phase boundary is at least as great as the dissipation rate during any "virtual motion" of that discontinuity whose speed $\dot{s}(t)$ at time $t$ coincides with that in the actual motion, but for which the virtual driving traction $f_{*}$ may take any value in the range allowed by the given $\dot{s}(t)$. This notion of maximum dissipation, which is easily seen to be equivalent to (5.16), is closely related to the concept of maximum plastic work utilized in the constitutive description of rate-independent elastic plastic solids; see the work of Lubliner [27] and Rice [33]. For a detailed discussion of the distinction between the two notions of maximum dissipation represented by the chord criterion and the entropy rate shock criterion, the reader is referred to [7].

Figure 13. kinetic relation $C$ corresponding to the chord criterion 
Acknowledgements. The authors have benefited from conversations with William Blackshaw, Richard James, James Rice and Phoebus Rosakis, to whom we express our thanks. We also thank Robert Pego and Michael Shearer for informative discussions concerning the "chord criterion", and Edward Zukoski for his helpful comments on deflagrations and detonations.

The support of the work summarized here by the Solid Mechanics Program of the Office of Naval Research through Contract N00014-87-K-0117 and Grant N00014-90-J-1871 is gratefully acknowledged.

The support and hospitality of the Institute for Mathematics and its Applications at the University of Minnesota during the Workshop on Shock-Induced Transitions and Phase Structures in General Media, October 15-19, 1990, and the associated funding from the National Science Foundation, are also acknowledged with thanks.

\section{REFERENCES}

[1] R. Abeyaratne, Discontinuous deformation gradients in the finite twisting of an elastic tube, Journal of Elasticity, 11 (1981), pp. 43-80.

[2] R. Abeyaratne and J.K. Knowles, On the dissipative response due to discontinuous strains in bars of unstable elastic material, International Journal of Solids and Structures, 24 (1988), pp. 1021-1044.

[3] Unstable elastic materials and the viscoelastic response of bars in tension, Journal of Applied Mechanics, 55 (1988), pp. 491-492.

[4] , On the driving traction acting on a surface of strain discontinuity in a continuum, Journal of the Mechanics and Physics of Solids, 38 (1990), pp. 345-360.

[5] Kinetic relations and the propagation of phase boundaries in solids, to appear in Archive for Rational Mechanics and Analysis.

[6] - Implications of viscosity and strain gradient effects for the kinetics of propagating phase boundaries in solids, to appear in SIAM Journal on Applied Mathematics.

[7] , On the propagation of maximally dissipative phase boundaries in solids, to appear in Quarterly of Applied Mathematics.

[8] H.B. CALLEN, Thermodynamics and an Introduction to Thermostatistics, second edition, Wiley, New York, 1985.

[9] J.W. Christian, The Theory of Transformations in Metals and Alloys, Part I, Pergamon, Oxford, 1975 .

[10] B.D. Coleman and M.E. GuRTin, Thermodynamics with internal state variables, Journal of Chemical Physics, 47 (1967), pp. 597-613.

[11] R. Courant and K.O. Friedrichs, Supersonic Flow and Shock Waves, Interscience, New York, 1948.

[12] C.M. Dafermos, Hyperbolic systems of conservation laws, in Systems of nonlinear partial differential equations, (J.M. Ball, editor), pp. 25-70, D. Riedel, Dordrecht, 1983.

[13] J.E. DUNn and J. SERRIN, On the thermomechanics of interstitial working, Archive for Rational Mechanics and Analysis, 88 (1985), pp. 95-133. 
[17] M. Grujicic, G.B. Olson and W.S. Owen, Mobility of martensite interfaces, Metallurgical Transactions A, 16A (1985), pp. 1713-1722.

[18] Mobility of the $\beta_{1}-\gamma_{1}^{\prime}$ martensitic interface in Cu-Al-Ni:PartI. Experimental measurements, Metallurgical Transactions A, 16A (1985), pp. 1723-1734.

[19] H. HAtToRi, The Riemann problem for a van der Waals fluid with entropy rate admissibility criterion. Isothermal case, Archive for Rational Mechanics and Analysis, 92 (1986), pp. $247-263$.

[20] The Riemann problem for a van der Waals fluid with entropy rate admissibility criterion. Nonisothermal case, Journal of Differential Equations, 65 (1986), pp. 158-174.

[21] W.D. Hayes, Gasdynamic Discontinuities, Princeton University Press, Princeton, 1960.

[22] R.D. JAMEs, The propagation of phase boundaries in elastic bars, Archive for Rational Mechanics and Analysis, 73 (1980), pp. 125-158.

[23] J.K. KNowles, On the dissipation associated with equilibrium shocks in finite elasticity, Journal of Elasticity, 9 (1979), pp. 131-158.

[24] D.J. KonTeWEg, Sur la forme que prennent les équations du mouvement des fluides si l'on tient compte des forces capillaires causées par des variation de densité, Archives Néerlandaises des Science Exactes et Naturelle, Series II, 6 (1901), pp. 1-24.

[25] R.V. Krishnan and L.C. Brown, Pseudo-elasticity and the strain-memory effect in an Ag-45 at. pct. Cd alloy, Metallurgical Transactions, 4 (1973), pp. 423-429.

[26] T.P. LiU, Uniqueness of weak solutions of the Cauchy problem for general $2 \times 2$ conservation laws, Journal of Differential Equations, 20 (1976), pp. 369-388.

[27] J. Lubliner, A maximum dissipation principle in generalized plasticity, Acta Mechanica, 52 (1984), pp. 225-237.

[28] O.A. OLEINIK, On the uniqueness of the generalized solution of the Cauchy problem for a nonlinear system of equations occurring in mechanics, Uspekhi Matematicheskii Nauk (N.S.), 12 (1957), pp. 169-176 (in Russian).

[29] Uniqueness and stability of the generalized solution of the Cauchy problem for a quasi-linear equation, Uspekhi Matematicheskii Nauk (N.S.), 14 (1959), pp. 165-170 (in Russian).

[30] R. PEgo, Phase transitions in one dimensional nonlinear viscoelasticity: admissibility and stability, Archive for Rational Mechanics and Analysis, 97 (1986), pp. 353-394.

[31] T.J. Pence, On the encounter of an acoustic shear pulse with a phase boundary in an elastic material: energy and dissipation, to appear in Journal of Elasticity.

[32] T. Poston and I.N. Stewart, Catastrophe Theory and its Applications, Pitman, London, 1978.

[33] J.R. RICE, On the structure of stress-strain relations for time-dependent plastic deformation in metals, Journal of Applied Mechanics, 37 (1970), pp. 728-737.

[34] , Inelastic constitutive relations for solids: An internal variable theory and its applications to metal plasticity, Journal of the Mechanics and Physics of Solids, 19 (1971), pp. $433-455$.

[35] Continuum mechanics and thermodynamics of plasticity in relation to microscale deformation mechanisms, in Constitutive Equations in Plasticity, (A.S. Argon, editor), pp. 23-79, MIT Press, Cambridge, Massachusetts, 1975.

[36] M. SHeARER, The Riemann problem for a class of conservation laws of mixed type, Journal of Differential Equations 46 (1982), pp. 426-443.

[37] , Nonuniqueness of admissible solutions of Riemann initial value problems for 
[40] , Dynamics of first order phase transitions, in Phase Transformations and Material Instabilities in Solids (M.E. Gurtin, ed.), pp. 163-203, Academic Press, New York, 1984 .

[41] , A limiting "viscosity" approach to the Riemann problem for materials exhibiting change of phase, Archive for Rational Mechanics and Analysis, 105 (1989), pp. 327-365.

[42] C. Truesdeld, Rational Thermodynamics, Springer-Verlag, New York, 1969.

[43] L. Truskinovsky, Equilibrium phase interfaces, Soviet Physics Doklady, 27 (1982), pp. $551-553$.

[44] Structure of an isothermal phase discontinuity, Soviet Physics Doklady, 30 (1985), pp. 945-948.

[45] , Dynamics of non-equilibrium phase boundaries in a heat-conducting non-linearly elastic medium, Journal of Applied Mathematics and Mechanics (PMM USSR), 51 (1987), pp. $777-784$. 